\title{
Comparação de estado nutricional e demais variáveis entre doentes renais agudos e crônicos hospitalizados
}

Comparison of nutritional status and other variables between hospitalized acute and chronic renal patients

DOI: $10.37111 /$ braspenj.2020352002

Patrícia Gonsalves de Deus

Simone Carla Benincá

Caryna Eurich Mazur ${ }^{3}$

\section{Unitermos:}

Antropometria. Estado Nutricional. Nefropatias. Hospitalização.

\section{Keywords:}

Anthropometry. Nutritional Status. Kidney Diseases. Hospitalization.

\section{Endereço de correspondência:}

Caryna Eurich Mazur

Universidade Estadual do Centro-Oeste - Nutrição

Rua Simeão Varela de Sá, 3 - Vila Carli Guarapuava, PR, Brasil

E-mail: carynanutricionista@gmail.com

\section{Submissão:}

3 de setembro de 2019

Aceito para publicação

21 de março de 2020

\section{RESUMO}

Introdução: A doença renal é compreendida por alterações na função renal, que se mantém por período variado, definindo-se de duas formas: insuficiência renal aguda (IRA) e doença renal crônica (DRC), ambas a depender do desenvolvimento da doença. $O$ objetivo deste estudo é identificar se há diferença do estado nutricional entre a IRA e DRC entre os gêneros e faixa etária dos pacientes hospitalizados e a prevalência de outras doenças associadas à doença renal. Método: Estudo prospectivo observacional. Foram selecionados 33 pacientes internados em um hospital de Guarapuava, Paraná. As informações coletadas foram avaliação antropométrica, recordatório alimentar de $24 \mathrm{~h}$, diagnóstico clínico e nutricional. Os resultados foram avaliados por análise descritiva e inferencial. Resultados: A média de idade foi de 61,0 $\pm 17,4$ anos, sendo a maioria do sexo feminino $(54,5 \% ; n=18)$ e idosos $(66,6 \% ; n=21)$. Quanto à classificação do estado nutricional, a maioria estava em eutrofia $(57,6 \%)$ e 42,4\% e 51,5\% estavam em desnutrição pela circunferência do braço e pela prega cutânea tricipital, respectivamente. Quanto aos antecedentes pessoais investigados, não foram encontradas diferenças estatisticamente significativas, havendo uma tendência estatística dos pacientes com diagnóstico clínico de DRC terem hipertensão arterial. Conclusão: Mesmo a maioria da população estudada apresentando estado nutricional adequado, observou-se tendência à desnutrição por meio de outros parâmetros de avaliação antropométrica.

\section{ABSTRACT}

Introduction: Kidney disease is comprised of changes in kidney function that is maintained for a varying period, defined in two ways: acute kidney failure (AKF) and chronic kidney disease (CKD), both depending on the development of the disease. The objective of this study is to identify whether there is a difference in the nutritional status between AKF and CKD between the genders and age groups of hospitalized patients and the prevalence of other diseases associated with kidney disease. Methods: Prospective observational study. We selected 33 patients admitted to a hospital in Guarapuava, Paraná. The collected information was anthropometric assessment, 24-hour food recall, clinical and nutritional diagnosis. The results were evaluated by descriptive and inferential analysis. Results: The mean age was $61.0 \pm 17.4$ years, with the majority being female $154.5 \%$; $\mathrm{n}=18$ ) and elderly $(66.6 \% ; \mathrm{n}=21)$, regarding the classification of nutritional status, most were eutrophic (57.6\%) and $42.4 \%$ and $51.5 \%$ were malnourished by the circumference of the arm and the tricipital skin fold, respectively. As for the personal history investigated, no statistically significant differences were found, with a statistical tendency for patients with a clinical diagnosis of CKD to have arterial hypertension. Conclusion: Even though the majority of the studied population had an adequate nutritional status, there was also a tendency towards malnutrition due to other parameters of anthropometric assessment.

1. Nutricionista, Pós-graduada em Nutrição Clínica no Centro Universitário Campo Real, Guarapuava, PR, Brasil.

2. Nutricionista, Doutora em Ciências da Saúde, Docente do Centro Universitário Campo Real, Guarapuava, PR, Brasil.

3. Nutricionista, Doutora em Medicina Interna, Docente do Curso de Nutrição. Universidade Estadual do Centro-Oeste (UNICENTRO), Guarapuava, PR, Brasil. 


\section{INTRODUÇÃO}

Os rins têm papel importante no equilíbrio hidroeletrolítico do organismo humano. A sobrevivência depende do funcionamento normal destes órgãos vitais. Os rins são responsáveis por eliminar toxinas do sangue por um sistema de filtração, regular a formação do sangue e ossos, regular a pressão sanguínea, e pelo controle do balanço químico e de líquidos do corpo'.

A doença renal é caracterizada por alterações na função renal, que se mantêm por período variado, definindo-se de duas formas: insuficiência renal aguda (IRA) e doença renal crônica (DRC), ambas a depender do desenvolvimento da doença a nível renal ${ }^{1,2}$.

A DRC é estabelecida como uma condição progressiva e irreversível das funções endócrinas, tubular e glomerular dos rins e tem como peculiaridade principal a diminuição do filtrado glomerular num período superior a três meses ${ }^{1,3}$. A perda progressiva contribui para o fracasso da capacidade do corpo em manter os equilíbrios metabólicos e hidroeletrolíticos, ocasionando retenção de ureia e demais resíduos nitrogenados no sangue ${ }^{4}$.

A IRA, também chamada de lesão renal aguda, é caracterizada pela perda repentina da capacidade dos rins em filtrarem resíduos, minerais e líquidos do sangue. Essa enfermidade é comum em pacientes hospitalizados com alguma outra condição. Pode desenvolver-se rapidamente, dentro de algumas horas, ou mais lentamente, durante alguns dias, podendo ser fatal e requerer tratamento intensivo. Entretanto, pode ser reversível; tudo depende do estado de saúde pregresso e atual do paciente ${ }^{1,4}$.

Fatores de risco considerados tradicionais, como hipertensão arterial, insuficiência cardíaca congestiva, dislipidemia, diabetes mellitus e tabagismo, podem ser consequências, pela alta mortalidade cardiovascular em pacientes renais ${ }^{5}$. Estudo prospectivo demonstrou que a combinação entre desnutrição, inflamação e aterosclerose (síndrome MIA) é comum em DRC e que seus componentes se relacionam entre si e com a alta mortalidade nesse grupo de pacientes ${ }^{5,6}$.

O paciente renal, especialmente o crônico, pode estar em risco nutricional devido ao alto catabolismo ou desnutrição energética-proteico associada à sarcopenia. Muitas vezes, há, também, restrição alimentar e hídrica, inflamações e infecções, além de alterações nos hormônios e sobrecarga cardiovascular, como comorbidades que reduzem a eficácia do tratamento clínico e nutricional e, consequentemente, a sobrevida destes indivíduos ${ }^{5,7,8}$.

Assim, o estado nutricional constitui um importante fator de risco clínico dessa população, sendo indispensável o diagnóstico nutricional com associação de fatores antropométricos, bioquímicos, clínicos e dietéticos dos pacientes, para adequar o tratamento, principalmente nos pacientes desnutridos, e prevenir a desnutrição nos eutróficos e com sobrepeso/obesidade?.

Diante da importância do estado nutricional no prognóstico do doente renal, esse trabalho tem como objetivo avaliar o estado nutricional desses pacientes no âmbito hospitalar, buscando relação com gênero e idade.

\section{MÉTODO}

Trata-se de um estudo prospectivo, realizado em um hospital em Guarapuava-PR, no período de julho de 2016 a fevereiro de 2017, que analisou pacientes portadores de doença renal, tanto IRA quanto DRC.

Esse estudo foi aprovado pelo Comitê de Ética em Pesquisa da Universidade Estadual do Centro-Oeste (UNICENTRO), sob o parecer número 1.593.833/2016.

Ressalta-se que os pacientes que concordaram em participar da pesquisa assinaram o Termo de Consentimento Livre e Esclarecido (TCLE), em duas vias, sendo uma delas entregue ao paciente, e a outra via arquivada com a pesquisadora responsável.

Foram inclusos, na pesquisa, pacientes entre 20 e 100 anos de idade, sem distinção de sexo, etnia ou estado nutricional. Foram excluídos do estudo, gestantes, crianças, pessoas que estavam na unidade de terapia intensiva (UTI) e aqueles que por algum motivo não se enquadravam nas características determinadas pelos critérios de inclusão propostos no estudo.

Ao final, a amostra foi então composta por 33 indivíduos, desses foram utilizadas as informações obtidas na avaliação antropométrica, tais como peso $(\mathrm{kg})$, altura $(\mathrm{m})$, índice de massa corporal atual (IMC) $\left(\mathrm{kg} / \mathrm{m}^{2}\right)$, circunferência do braço $(C B)$, e circunferência de panturrilha (CP). O IMC foi calculado utilizando o peso do indivíduo dividido pela altura ao quadrado e foram classificados em: magreza $>$ $18,5 \mathrm{~kg} / \mathrm{m}^{2}$, eutrofia IMC > 18,50 a 24,99 kg/m², sobrepeso IMC $\geq 25$ a $29,99 \mathrm{~kg} / \mathrm{m}^{2}$ e obesidade IMC $\geq 30 \mathrm{~kg} /$ $\mathrm{m}^{2}{ }^{10}$. A classificação do IMC de idosos, segundo Lipschitz ${ }^{11}$, foi: magreza $<22$, eutrofia de 22 a 22 e $>27$ excesso de peso. A CB foi classificada em desnutrição, eutrofia, sobrepeso e obesidade, de acordo com adequação proposta por Blackburn e Thornton'2. A CP foi classificada de acordo com Bonnefoy et al. ${ }^{13}$, valores menores de $30,5 \mathrm{~cm}$ indicaram perda de massa muscular.

Para análise estatística foram considerados valores descritivos, como frequências absolutas e relativas, além de médias e desvios padrões. Para avaliação da normalidade da amostra foi calculado o teste de Shapiro Wilk. Para comparação de dados paramétricos foi utilizado o teste T de Student, enquanto para dados não-paramétricos foi utilizado o teste de qui-quadrado de Fisher. Foram considerados significantes 
quando os valores de p foram menores que 0,05. A análise foi realizada com auxílio do software SPSS ${ }^{\circledR}$ versão 22.0 .

\section{RESULTADOS}

Dentre os 33 pacientes foi constatada média de idade de $61,0 \pm 17,4$ anos, sendo a maioria do sexo feminino $(54,5 \% ; n=18)$ e idosos $(66,6 \% ; n=21)$. Conforme observado na Tabela 1, entre as duas classificações de doença renal, a DRC foi a mais prevalente $(57,6 \% ; n=19)$, dentre esses pacientes, a maioria apresentava hipertensão arterial $(66,7 \% ; n=22)$.

A média do IMC, no geral, foi de 23,9 $\pm 4,2 \mathrm{~kg} / \mathrm{m}^{2}$, a média de CB 27,9 $\pm 4,4 \mathrm{~cm}$, a média da PCT 11,7 $\pm 6,6$ $\mathrm{mm}$, a média de CMB foi $22,3 \pm 8,4 \mathrm{~cm}$ e a $C P$ média foi $32,9 \pm 5,3 \mathrm{~cm}$. Quanto à classificação, a maioria estava em eutrofia pelo IMC $(57,6 \%)$ e CMB $(66,7 \%)$, e em desnutrição pela CB $(42,4 \%)$ e PCT $(51,5 \%)$ (Tabela 2$)$.

De acordo com os resultados demonstrados na Tabela 3 , não foram encontradas diferenças estatisticamente significativas, havendo tendência estatística dos pacientes com DRC terem o diagnóstico de hipertensão arterial sistêmica. Quanto à aceitação da dieta, observou-se que $57 \%$ dos pacientes com DRC não aceitaram bem a dieta, já os com IRA foi equiparada ( $50 \%$ aceitação boa e $50 \%$ aceitação ruim).

\begin{tabular}{|c|c|c|}
\hline Variáveis & $\mathrm{n}$ & $\%$ \\
\hline \multicolumn{3}{|l|}{ Diagnóstico Clínico } \\
\hline Insuficiência renal aguda & 14 & 42,4 \\
\hline Doença renal crônica & 19 & 57,6 \\
\hline \multicolumn{3}{|l|}{ Sexo } \\
\hline Feminino & 18 & 54,5 \\
\hline Masculino & 15 & 45,5 \\
\hline \multicolumn{3}{|l|}{ Anos de estudo } \\
\hline$\leq 8$ anos & 14 & 42,4 \\
\hline$>8$ anos & 19 & 57,6 \\
\hline \multicolumn{3}{|l|}{ Antecedentes pessoais } \\
\hline Dislipidemias & 8 & 24,2 \\
\hline Hipertensão arterial & 22 & 66,7 \\
\hline Diabetes mellitus & 9 & 27,3 \\
\hline Câncer & 3 & 9,1 \\
\hline Tabagismo & 8 & 24,2 \\
\hline Etilismo & 4 & 12,1 \\
\hline \multicolumn{3}{|l|}{ Prescrição dietoterápica } \\
\hline Livre & 13 & 39,4 \\
\hline Branda & 6 & 18,6 \\
\hline Leve & 8 & 24,2 \\
\hline Outras & 6 & 18,6 \\
\hline \multicolumn{3}{|l|}{ Aceitação da dieta } \\
\hline Boa & 15 & 45,5 \\
\hline Ruim & 18 & 54,5 \\
\hline
\end{tabular}

Tabela 2 - Estado nutricional dos pacientes conforme o diagnóstico clínico.

\begin{tabular}{|c|c|c|c|c|}
\hline Variáveis & $\begin{array}{c}\text { Total } \\
(\mathrm{n}=33)\end{array}$ & $\begin{array}{c}\text { IRA } \\
(n=14)\end{array}$ & $\begin{array}{c}\text { DRC } \\
(n=19)\end{array}$ & $\begin{array}{l}\text { Valor } \\
\text { de } p\end{array}$ \\
\hline Idade (anos) - média/DP & $61,0 \pm 17,4$ & $58,3 \pm 18,7$ & $63,0 \pm 16,6$ & $0,45^{\star}$ \\
\hline IMC $\left(\mathrm{kg} / \mathrm{m}^{2}\right)$ - média/DP & $23,9 \pm 4,2$ & $23,9 \pm 4,9$ & $24,0 \pm 3,7$ & $0,96^{*}$ \\
\hline \multicolumn{5}{|l|}{ Classificação IMC (\%; n) } \\
\hline Baixo peso & $9,1(3)$ & $14,3(2)$ & $5,3(1)$ & \multirow{3}{*}{$0,61^{\star \star}$} \\
\hline Eutrofia & $57,6(19)$ & $64,3(9)$ & $52,6(10)$ & \\
\hline Excesso de peso/ obesidade & $33,3(11)$ & $21,4(3)$ & $42,1(8)$ & \\
\hline CB (cm) - média/DP & $27,9 \pm 4,4$ & $27,3 \pm 4,9$ & $28,3 \pm 4,0$ & $0,52^{*}$ \\
\hline \multicolumn{5}{|l|}{ Classificação CB (\%; n) } \\
\hline Desnutrição & $42,4(13)$ & $50,0(7)$ & $31,6(6)$ & \multirow[t]{3}{*}{$0,65^{\star \star}$} \\
\hline Eutrofia & $33,3(11)$ & $28,6(4)$ & $36,8(7)$ & \\
\hline Sobrepeso/obesidade & $27,3(9)$ & $21,4(3)$ & $31,6(6)$ & \\
\hline PCT (mm) - média/DP & $11,7 \pm 6,6$ & $12,8 \pm 7,4$ & $10,8 \pm 6,0$ & $0,44^{*}$ \\
\hline \multicolumn{5}{|l|}{ Classificação PCT (\%; n) } \\
\hline Desnutrição & $51,5(17)$ & $57,1(8)$ & $47,4(9)$ & \multirow[t]{3}{*}{$0,26^{\star \star}$} \\
\hline Eutrofia & $15,2(5)$ & $14,3(2)$ & $15,8(3)$ & \\
\hline Sobrepeso/obesidade & $33,3(11)$ & $28,6(4)$ & $36,8(7)$ & \\
\hline CMB (cm) - média/DP & $22,3 \pm 8,4$ & $21,9 \pm 7,9$ & $22,7 \pm 8,9$ & $0,79^{\star}$ \\
\hline \multicolumn{5}{|l|}{ Classificação CMB (\%; n) } \\
\hline Desnutrição & $33,3(11)$ & $28,6(4)$ & $36,8(7)$ & \multirow[t]{2}{*}{$0,86^{\star \star}$} \\
\hline Eutrofia & $66,7(22)$ & $71,4(10)$ & $63,2(12)$ & \\
\hline $\mathrm{CP}(\mathrm{cm})$ - média/DP & $32,9 \pm 5,3$ & $32,6 \pm 5,3$ & $33,2 \pm 5,4$ & $0,81^{*}$ \\
\hline \multicolumn{5}{|l|}{ Classificação CP (\%; n) } \\
\hline Perda de massa magra & $27,3(9)$ & $21,4(3)$ & $31,6(6)$ & \multirow[t]{2}{*}{$0,63^{* *}$} \\
\hline Sem perda de massa magra & $72,7(24)$ & $78,6(11)$ & $68,4(13)$ & \\
\hline -quadrado de Fisher. & $\mathrm{PP}=$ desvio $-\mathrm{p}$ & drão; $\mathrm{cm}=\mathrm{ce}$ & metros; mm & illime \\
\hline
\end{tabular}

Tabela 3 - Dieta e fatores associados aos pacientes conforme o diagnóstico clínico.

\begin{tabular}{|c|c|c|c|}
\hline Variáveis & IRA $(n=14)$ & DRC $(n=19)$ & Valor de $p$ \\
\hline \multicolumn{4}{|l|}{ Sexo (\%; n) } \\
\hline Feminino & $71,4(10)$ & $42,1(8)$ & 0,09 \\
\hline Masculino & $28,6(4)$ & $57,9(11)$ & \\
\hline \multicolumn{4}{|l|}{ Anos de estudo (\%; n) } \\
\hline$\leq 8$ anos & $28,6(4)$ & $52,6(10)$ & 0,04 \\
\hline$>8$ anos & $71,4(10)$ & $47,4(9)$ & \\
\hline \multicolumn{4}{|c|}{ Antecedentes pessoais (\%; n) } \\
\hline Dislipidemias & $28,6(4)$ & $21,0(4)$ & 0,62 \\
\hline Hipertensão arterial & $50,0(7)$ & $78,9(15)$ & 0,07 \\
\hline Diabetes mellitus & $14,3(2)$ & $36,8(7)$ & 0,15 \\
\hline Câncer & - & $15,8(3)$ & 0,08 \\
\hline \multicolumn{4}{|c|}{ Prescrição dietoterápica (\%; n) } \\
\hline Livre & $50,0(7)$ & $31,6(6)$ & \\
\hline Branda & $14,3(2)$ & $21,0(4)$ & 0,35 \\
\hline Leve & $35,7(5)$ & $15,8(3)$ & \\
\hline Outras & - & $31,6(6)$ & \\
\hline \multicolumn{4}{|c|}{ Aceitação da dieta (\%; n) } \\
\hline Boa & $50,0(7)$ & $42,1(8)$ & 0,33 \\
\hline Ruim & $50,0(7)$ & $57,9(11)$ & \\
\hline
\end{tabular}




\section{DISCUSSÃO}

Esse estudo é um importante instrumento da literatura para compreender as diferenças, em termos nutricionais, de pacientes hospitalizados com diagnósticos clínicos de DRC e IRA. Trata-se de uma pesquisa que pode subsidiar a conduta nutricional para as diferentes situações clínicas, especialmente nas dimensões antropométricas/corporais, de doentes renais que necessitam de hospitalização.

Os pacientes estudados foram separados em doentes renais crônicos e agudos, a maior parte da amostra foi composta por pacientes do sexo feminino. Dados parecidos com um estudo realizado com pacientes diagnosticados com IRA, no município de Guarapuava, onde 58,7\% da amostra estudada eram do sexo masculino e $42,3 \%$ do feminino ${ }^{14}$. Observou-se que a média de idade da população estudada é de aproximadamente 61 anos, diferente da pesquisa de Souza et al. ${ }^{15}$, na qual a média de idade da amostra foi de 47,3 anos. Em outro estudo desenvolvido com 96 indivíduos, que avaliou o estado nutricional de pacientes com DRC em hemodiálise, internados em hospitais públicos, a média de idade foi de 54 anos$^{9}$. Evidenciando, portanto, uma população relativamente idosa comparadas aos demais estudos presentes na literatura.

No presente estudo, observou-se que, dentre os pacientes avaliados classificados eutróficos não houve diferença entre a DRC e a IRA. Sabe-se que a utilização de parâmetros antropométricos, como a utilização de circunferências é importante para o estabelecimento do diagnóstico nutricional. Foi possível observar pela CB e PCT que a maioria dos pacientes avaliados foi classificada como desnutrida, e não apresentou perda de massa magra pela $\mathrm{CP}$, não houve diferença quando comparadas a DRC e IRA. Em uma pesquisa realizada com 58 pacientes em tratamento dialítico no nordeste do Brasil, foi encontrada por meio da avaliação antropométrica, 12,1\% de desnutrição pelo IMC, 84,5\% da PCT, e 43\% da CMB, diferente portanto do presente trabalho ${ }^{16}$. Já em estudo desenvolvido por Santos et al. ${ }^{9}, 14,6 \%$ da população apresentaram desnutrição, 33,3\% eutrofia e 52,1\% tinham ao menos uma variável relacionada à desnutrição. É preciso reiterar que, muitas vezes, é necessária a utilização de vários instrumentos de avaliação nutricional para adequada compreensão do estado global do indivíduo, especialmente do paciente renal.

Sabe-se que os graus de desnutrição na DRC relatados na literatura variam amplamente e mesmo os indivíduos com sobrepeso ou obesidade apresentam risco de desnutrição proteica, apesar do hipercatabolismo da doença e ingestão calórica aumentada ${ }^{17}$. É conhecido que o estado nutricional depletado no começo do tratamento dialítico contribui para pior sobrevida ao decorrer dos anos. A causa da desnutrição energético proteica é associada, na maioria das vezes, à baixa ingestão alimentar ou ao hipercatabolismo ${ }^{18}$.
Entretanto, não foi possível a estimativa de gasto energético desses pacientes devido à falta de equipamentos para tal. O presente estudo demonstrou que, dentre os pacientes avaliados, $50 \%$ aceitaram bem a dieta proposta no hospital. Dados como esse são importantes para denotar a qualidade do serviço de nutrição e dietética hospitalar e traçar propostas dietoterápicas mais atraentes para melhoria do quadro clínico dos pacientes hospitalizados.

Quando analisada a doença renal associada aos antecedentes pessoais do individuo, observou-se que $66,7 \% \mathrm{da}$ amostra eram hipertensos. Ao serem comparadas distintamente, a DRC $(78,9 \%)$ apresentou maior prevalência em relação à IRA (50\%). Dados encontrados na literatura relativos às doenças associadas foram de maior percentual, cerca de $53,3 \%$ hipertensos referentes à DRC ${ }^{19}$. Os doentes renais crônicos em diálise também costumam ter a hipertensão como comorbidade (cerca de 60\% a $80 \%$ ), com um complicado controle da doença devido às alterações do sistema renina-angiotensina-aldosterona, aumento da atividade simpática e outras etiologias ${ }^{20}$. Por fim, ao compará-las entre elas, DRC e IRA, não houve diferença estatística significativa, porém na literatura observa-se que, tanto a DRC e a IRA, apresentam grande prevalência para a hipertensão arterial. Ainda, foi possível observar que os pacientes com diagnóstico de DRC possuíam menor tempo de estudo quando comparados aos pacientes com IRA, o que pode estar associado à etiologia da própria doença.

Como limitações desse trabalho estão a falta de acesso à informação sobre o tratamento clínico desses pacientes, assim como a não avaliação do consumo alimentar em termos quali-quantitativos.

\section{CONCLUSÃO}

Com base nos resultados, conclui-se que, mesmo a maioria da população estudada apresentando estado nutricional adequado por meio do IMC, observou-se tendência à desnutrição por meio de outros parâmetros de avaliação antropométrica. Assim, fica clara a importância do acompanhamento nutricional na prevenção da desnutrição ou do excesso de peso, nos pacientes com DRC ou IRA. Referente aos antecedentes pessoais, nota-se que a maior parte dos pacientes apresentava comorbidades associadas, dentre elas a hipertensão arterial apresentou maior prevalência.

\section{REFERÊNCIAS}

1. Thomé FS, Sesso RC, Lopes AA, Lugon JR, Martins CT. Inquérito brasileiro de diálise crônica 2017. J Bras Nefrol. 2019;41(2):208-14.

2. Daugirdas JT, Blake PG, Ing TS. Manual de diálise. $3^{\text {a }}$ ed. Rio de Janeiro: Medsi; 2003.

3. Santos RLG, Oliveira DRF, Nunes MGS, Barbosa RMP, Gouveia VA. Avaliação do conhecimento do paciente renal crônico 
em tratamento conservador sobre modalidades dialíticas. Rev enferm UFPE on line. 2015;9(2):651-60.

4. Brasil. Ministério da Saúde. Secretaria de Atenção à Saúde. Departamento de Atenção Especializada e Temática. Diretrizes clínicas para o cuidado ao paciente com doença renal crônica DRC no Sistema Único de Saúde. Brasília: Ministério da Saúde; 2014.

5. Allawi AAD. Malnutrition, inflamation and atherosclerosis (MIA syndrome) in patients with end stage renal disease on maintenance hemodialysis (a single centre experience). Diabetes Metab Syndr. 2018;12(2):91-7.

6. Pecoits-Filho R, Stevinkel P, Lindholm B, Bergstrom J, Noronha I, Abensur H. Revisão: desnutrição, inflamação e aterosclerose (síndrome MIA) em pacientes portadores de insuficiência renal crônica. J Bras Nefrol. 2002;24(3):136-46.

7. Souza VA, Oliveira D, Mansur HN, Fernandes NMS, Bastos MG. Sarcopenia na doença renal crônica. J Bras Nefrol. 2015;37(1):98-105.

8. Costa LG. Caracterização e estado nutricional de portadores de insuficiência renal crônica em tratamento hemodialítico no Distrito Federal [Dissertação de Mestrado]. Brasília: Universidade de Brasília; 2015.

9. Santos KB, Costa LG, Andrade JML. Estado nutricional de portadores de doença renal crônica em hemodiálise no Sistema Único de Saúde. Ciência \& Saúde Col. 2019;24(3):1189-99.

10. World Health Organization. Obesity: preventing and managing the global epidemic. Geneva: World Health Organization; 2000. [cited 2020 Aug 21]. Available from: https://www.who.int/ nutrition/publications/obesity/WHO_TRS_894/en/
11. Lipschitz DA. Screening for nutritional status in the elderly. Primary Care. 1994;21(1):55-67.

12. Blackburn GL, Thornton PA. Nutritional assessment of the hospitalized patient. Med Clin North Am. 1979;63(5):11103-15.

13. Bonnefoy M, Jauffret M, Kostka T, Jusot JF. Usefulness of calf circumference measurement in assessing the nutritional state of hospitalized elderly people. Gerontology. 2002;48(3):162-9.

14. Pivatto DR, Abreu IS. Principais causas de hospitalização de pacientes em hemodiálise no município de Guarapuava, Paraná, Brasil. Rev Gaúcha Enferm. 2010;31(3):515-20.

15. Souza MVS, Silva JB, Mesquita VJP, Franco LMN, Costa MA, Pereira ALS, et al. Avaliação do estado nutricional e da adequação alimentar de pacientes em hemodiálise em um hospital regional do Distrito Federal. REAS. 2019;11(1):e109.

16. Oliveira CMC, Kubrusly M, Mota RS, Silva CAB, Oliveira VN. Desnutrição na insuficiência renal crônica: qual o melhor método diagnóstico na prática clínica? J Bras Nefrol. 2010;32(1):57-70.

17. Stolic R. Obesity in renal failure: health or disease? Med Hypotheses. 2010;75(6):497-500.

18. Cuppari L, Kamimura MA. Avaliação nutricional na doença renal crônica: desafios na prática clínica. J Bras Nefrol. 2009;31(supl. 1)S1:28-35.

19. Santos ACB, Machado MC, Pereira LR, Abreu JLP, Lyra MB. Association between the level of quality of life and nutritional status in patients undergoing chronic renal hemodialysis. J Bras Nefrol. 2013;35(4):279-88.

20. Pinheiro ME, Alves CMP. Hipertensão arterial na diálise e no transplante renal. J Bras Nefrol 2003;25(3):142-8.

Local de realização do estudo: Centro Universitário Campo Real, Guarapuava, PR, Brasil.

Conflito de interesse: Os autores declaram não haver. 